\title{
New developments in the endoscopic surveillance of Barrett's oesophagus
}

\author{
J J G H M Bergman, G N J Tytgat
}

Gut 2005;54(Suppl I):i38-i42. doi: 10.1136/gut.2004.041590

Patients with a Barrett's oesophagus are at risk for developing an adenocarcinoma of the distal oesophagus. Therefore, many patients undergo endoscopic surveillance to detect dysplasia and/or cancer at an early and curable stage. However, early neoplastic lesions are difficult to identify with standard endoscopy. In addition, the low incidence of these lesions, currently estimated at $0.5 \%$ per year, reduces the cost effectiveness of the surveillance strategy. New developments, aimed at improving the efficacy of Barrett's surveillance, focus on two areas: 1) improvement of the endoscopic detection of early neoplastic lesions; and 2) the use of alternative techniques for tissue sampling combined with molecular markers to identify patients at risk for malignant degeneration.

See end of article for authors' affiliations

Correspondence to: Dr J J G H M Bergman, Department of Gastroenterology, Academic Medical Center, Meibergdreef 9, 1105 AZ Amsterdam, Netherlands; i.j.bergman@amc.uva.nl
B arrett's oesophagus is a condition in which the normal squamous lining of the distal oesophagus has been replaced by columnar epithelium with intestinal metaplasia. ${ }^{1}$ Barrett's oesophagus is considered to be a premalignant condition that predisposes for oesophageal adenocarcinoma. ${ }^{1}$ Malignant degeneration of Barrett's metaplasia is thought to be a multistep process in which intestinal metaplasia progresses through low grade and high grade dysplasia into eventually invasive cancer. ${ }^{2}$ Most international gastroenterological societies therefore advise endoscopic surveillance of patients with a known Barrett's oesophagus in order to detect cancer and its precursor lesions at an early and curable stage. ${ }^{3}$ In this article we focus on new techniques to improve the quality of the endoscopic surveillance of Barrett's patients.

\section{THE CURRENT STATUS OF BARRETT'S SURVEILLANCE AND ITS PROBLEMS}

The presence of a Barrett's oesophagus can be suspected at endoscopy if a salmon coloured epithelium is seen above the proximal end of the gastric folds, thus indicating the presence of metaplastic columnar epithelium. Histopathologically there are three types of metaplastic columnar epithelium: gastric fundus type, gastric cardia type, and intestinal type. Because only the intestinal type is considered to bear malignant potential, the presence of intestinal metaplasia is increasingly considered to be a prerequisite for the diagnosis of Barrett's oesophagus. ${ }^{4}$ The Barrett's mucosa is often very heterogeneous of composition: areas fundic/ cardiac metaplasia, intestinal metaplasia as well as low grade dysplasia, high grade dysplasia, and early cancer can all be present simultaneously and are difficult to distinguish with standard endoscopy. ${ }^{5}$ This poses one of the main problems for the efficacy of Barrett's surveillance: early neoplastic lesions are difficult to identify. In the absence of visible abnormalities, therefore, four quadrant biopsies are taken for every $2 \mathrm{~cm}$ length of Barrett's epithelium. ${ }^{6}$ Apart from being time consuming and uncomfortable for the patient this is inevitably associated with sampling error. Another problem in the surveillance of Barrett's patients is the high intra- and interobserver variability in the histological grading of the biopsies, especially for the diagnosis low grade dysplasia. ${ }^{7}$ Finally the incidence of high grade dysplasia and early cancer, currently estimated at $0.5 \%$ per year, ${ }^{1}$ reduces the cost effectiveness of any surveillance strategy: the vast majority of Barrett's patients will never develop oesophageal cancer but currently still are candidates for an expensive and labour intensive surveillance programme. New developments, aimed at improving the efficacy of Barrett's surveillance, focus on two areas: 1) improvement of the endoscopic detection of early neoplastic lesions (thus enabling prolongation of the surveillance interval without increasing the incidence of interval cancers; and 2) the use of alternative techniques for tissue sampling combined with molecular markers to identify patients at risk for malignant degeneration.

In this article we give an overview of new developments that may improve the endoscopic detection of early Barrett's neoplasia and discuss the use of molecular markers for this indication.

\section{HOW TO IMPROVE THE ENDOSCOPIC DETECTION OF EARLY NEOPLASIA IN BARRETT'S OESOPHAGUS}

Standard video endoscopes have a focal distance between $\mathrm{l}$ and $2 \mathrm{~cm}$ from the tip of the endoscope and use less than 200000 pixels to construct an image. This limits the possibilities for detailed inspection of the mucosa; if the endoscope is moved too close to an area of interest the image becomes blurred ("out of focus"), whereas if the image is electronically enlarged ("digital zoom") the number of pixels and thus image resolution becomes reduced.

Abbreviations: CCD, charge coupled device; FISH, fluorescent in situ hybridisation; LIFE, light induced fluorescence endoscopy; LIFS, light induced fluorescence spectroscopy; $\mathrm{NBI}$, narrow band imaging; OCT, optical coherence tomography 
High resolution endoscopes with high quality charge coupled device (CCD) chips (>850 000 pixels) and variable focal distance are now commercially available. These endoscopes have a mechanically or electronically moveable lens at the distal tip that enables the endoscopist to zoom in onto an area of interest. Recent studies have shown that high resolution endoscopy can adequately distinguish areas of intestinal metaplasia from areas with gastric mucosa. ${ }^{8}$ Sharma et al reported that areas with high grade dysplasia were characterised by an irregular mucosal pattern enabling their detection with high resolution endoscopy. ${ }^{10}$ The detection of early mucosal abnormalities can be further enhanced by the use of staining agents such as methylene blue and indigo carmine. Methylene blue is a vital stain that is taken up by actively absorbing epithelial cells, which leads to specific staining of areas with intestinal metaplasia. Although randomised studies have shown that methylene blue staining increases the detection of dysplasia and early cancer in Barrett's oesophagus, ${ }^{11}$ others have found it to be disappointing. ${ }^{12}$ We have found that methylene blue staining is time consuming and operator dependent and therefore prefer to combine high resolution endoscopy with indigo carmine as a contrast staining agent (fig 1). ${ }^{10}$

The mucosal detail as seen with high resolution endoscopy can be further increased by using so called narrow band imaging (NBI). The NBI system has a standard high resolution mode in which the white light image is composed of the sequential imaging through a red, green, and blue band pass filter. In the NBI mode, the bandwidths of these three filters have been narrowed and the relative contribution of the blue filter has been increased resulting in improved mucosal contrast and detail. The endoscopist can switch back and forth between the high resolution mode and the NBI mode (fig 2). This technique holds the promise of obtaining images comparable to high resolution endoscopy with chromoscopy but without the use of dyes. ${ }^{13}$

Fluorescence endoscopy involves stimulation of certain molecules-fluorophores-by ultraviolet or blue light. Upon excitation, these fluorophores emit fluorescent light spread over a range of longer wavelengths from the green to the red spectrum. This is called autofluorescence and responsible endogenous fluorophores include collagen, NADH, aromatic amino acids, and porphyrins, each having their own excitation and emission wavelength. Normal metaplastic and dysplastic tissue have different autofluorescence spectra because of various compositions of fluorophores.
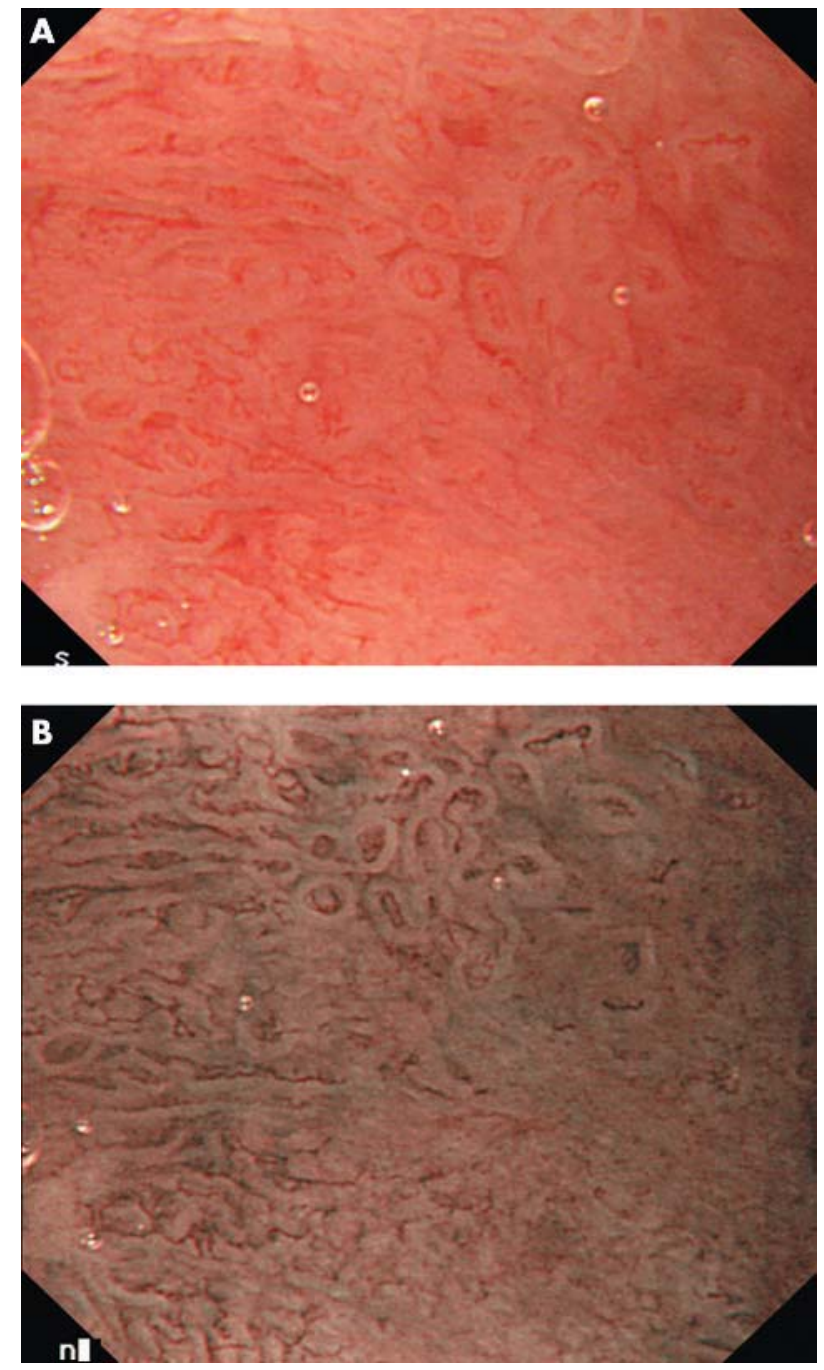

Figure 2 (A) High resolution endoscopy of a Barrett's oesophagus showing an area with an irregular mucosal pattern corresponding to high grade dysplasia; (B) high resolution endoscopy with narrow band imaging (NBI) of the same area. In NBI, the bandwidths of the standard red, green, and blue pass filters have been narrowed and the relative contribution of the blue filter has been increased resulting in improved mucosal contrast and detail.

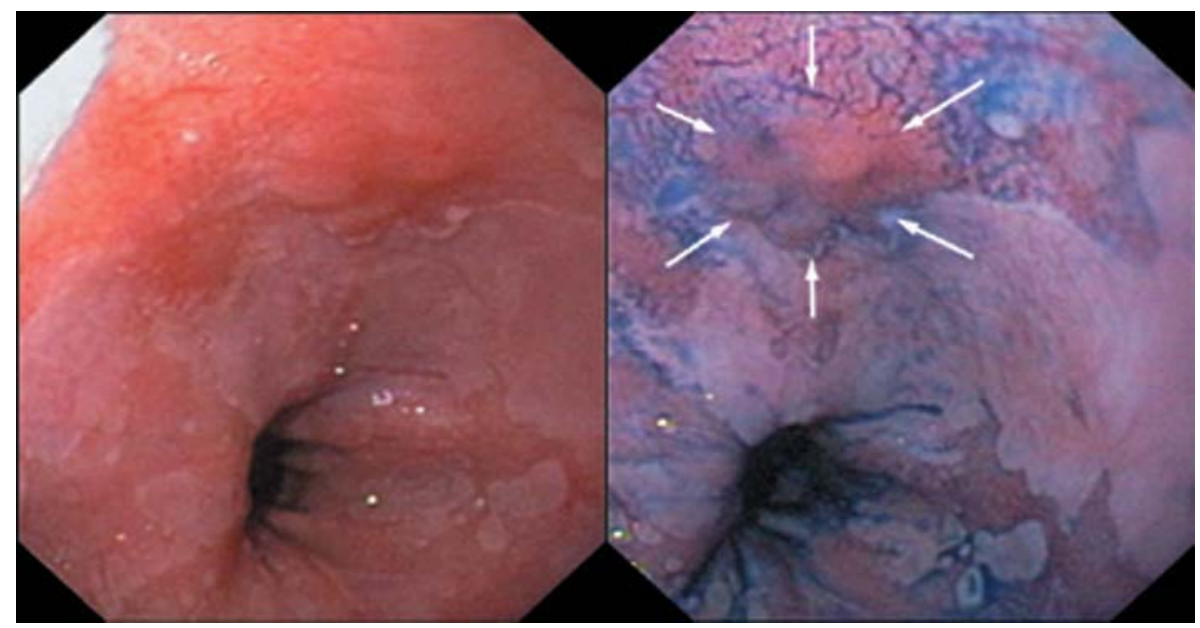

Figure 1 (A) Barrett's oesophagus with a small inconspicuous area of high grade dysplasia; (B) after staining with indigo carmine the lesion is much easier identified. 
Autofluorescence is also affected by tissue morphology such as mucosal thickening and irregularity, mainly because it attenuates the strong autofluorescence of the submucosal collagen. In addition, autofluorescence is affected by the presence of substances that absorb the emitted fluorescent light: haemoglobin being the most important. In light induced fluorescence spectroscopy (LIFS), small probes, passed through the working channel of an endoscope, are used to send the excitation light on to the tissues and to collect the emitted fluorescent light. These fluorescence spectra can then be analysed statistically. The value of LIFS in detecting dysplasia in Barrett's patients has been clinically evaluated by several groups with excellent results in distinguishing non-dysplastic areas from areas with high grade dysplasia and/or cancer. ${ }^{14}{ }^{15}$ The problem with LIFS is that it only samples a small mucosal area (1-3 mm), which makes this technique impractical for scanning larger areas. The technique has therefore been incorporated in a fibre optic endoscopic system-that is, light induced fluorescence endoscopy (LIFE). This system has both a standard white light mode and an autofluorescence mode. The autofluorescence mode consists of a mercury light source with a blue light pass filter (wave length $437 \mathrm{~nm}$ ), a camera equipped with two high sensitivity image sensors for detection of green (500-550 nm) and red (630-650 nm) autofluorescence, and an image processor for displaying the autofluorescence image. The input from these two sensors is subsequently used to construct a real time pseudocolour image where dysplastic lesions are displayed as dark red and normal tissue as light blue. Initial studies suggested that LIFE increased the detection rate of high grade dysplasia and cancer of standard endoscopy.$^{16}$ In a recent randomised crossover study comparing LIFE with standard endoscopy, LIFE did not improve the detection of high grade dysplasia or early cancer. ${ }^{17}$ The problem with the currently available fluorescence endoscopy system relates to the use of a fibre optic endoscope, which produces images inferior to those obtained by standard or high resolution endoscopy. In addition, the algorithms used to construct the fluorescence image are still rather primitive. A third generation fluorescence endoscope has now been developed that uses two separate CCD chips for both standard (video) endoscopic imaging and autofluorescence imaging. In addition, the autofluorescence image is constructed by using both the fluorescence and the reflectance characteristics of the tissue, which may further enhance the specificity of this technique.

Optical coherence tomography (OCT) is a technique that uses infrared light for excitation after which the reflected light is analysed for its delay and intensity of reflection. ${ }^{18}$ The images are obtained real time by a miniprobe that is advanced through the working channel of the endoscope. The basic principles of this technique and the aspect of the images obtained are comparable with those of high frequency miniprobe endosonography but instead of sound waves, OCT utilises infrared light. The in vivo resolution is approximately $10 \mu \mathrm{m}$, which results in images that strongly resemble those of histological microscopy of the superficial oesophageal wall layers. In our opinion it is questionable if such a real time full pseudo-histological investigation of the distal oesophagus during an endoscopic procedure is feasible at this point. The current systems obtain four "pseudo-histological" images per second and interpretation is hampered by difficulty in maintaining optimal focal distance because of movements by peristalsis, heartbeat, and respiration. Furthermore, image resolution is still insufficient to compete with standard microscopic histological images. Moreover, endoscopists are usually not trained in interpreting such images. Finally, even under optimal circumstances, pathologists have difficulty in evaluating dysplasia. A higher rate of image acquisition combined with an automated image analysis technique will be necessary in the future to justify OCT as a valid technique for surveillance of Barrett's patients.

Other imaging techniques, such as light scattering spectroscopy and Raman spectroscopy, utilise subtle changes in wave length that occur after the non-elastic interaction of light photons with cellular structures and molecular vibrations, respectively. The technical background of these experimental techniques is beyond the focus of this article, yet the information obtained may be very specific. ${ }^{19}$ Currently, these techniques are only available as spectroscopy techniques that use probes that sample a very small surface area of the mucosa. Incorporating these techniques in a wide angle real time endoscopic system will be technically challenging.

\section{NEW DEVELOPMENTS IN THE ENDOSCOPIC SURVEILLANCE OF BARRETT'S OESOPHAGUS: ARE THEY CLINICALLY RELEVANT?}

It is important to realise that although important steps have been made to improve the endoscopic imaging of patients with a Barrett's oesophagus, many questions remain unanswered. Most of these techniques are only available as prototypes, which limits their availability and leaves their cost effectiveness unclear. In addition, these techniques are usually tested in centres with both a high volume of relevant patients as well as experienced endoscopists. Usually there are no intra- or inter-observer studies available, nor have these systems been tested in patient populations with a much lower risk of early neoplasia than those referred to expert centres.

The incidence of high grade dysplasia and early cancer in patients with a Barrett's oesophagus is currently estimated at $0.5 \%$ per year and the cost effectiveness of any surveillance strategy has been questioned. The vast majority of Barrett's patients will never develop oesophageal cancer, thus including them in an expensive and labour intensive endoscopy programme using high tech imaging techniques is even more questionable. Hopes are set therefore on the detection of molecular markers to identify those patients who are at risk for malignant degeneration.

\section{MOLECULAR MARKERS AND ALTERNATIVE TISSUE SAMPLING TECHNIQUES \\ Molecular markers}

Apart from the cellular and architectural morphological changes, a wide range of molecular abnormalities-both phenotypic and genotypic-occur during the process of malignant degeneration in Barrett's metaplasia. ${ }^{20}$ Phenotypic changes encompass, amongst others, increased proliferation (marker: Ki-67 ${ }^{21}$ ), increased expression of growth factors (EGF, c-erbB2, and TGF- $\alpha^{2}$ ), increased expression of inflammatory factors (COX-2,,$\left.^{22} \mathrm{TNF}-\alpha^{23}\right)$, and disturbed cell adhesion (reduced expression of E-cadherine ${ }^{20}$ ). In addition, multiple genetic abnormalities have been reported: apart from gross chromosomal abnormalities, specific mutationsin the tumour suppression genes p53 and pl6-have been described. Finally, changes in the cell cycle of the involved epithelium, with an increased number of cells in the S phase (corresponding to DNA synthesis) and the G2 phase (premitosis), have been described..$^{24}$

Because several of these markers precede the morphological changes required for the histological diagnosis dysplasia, they can be used as markers-that is, molecular markers, to identify patients at risk for malignant degeneration. Unfortunately none of the investigated markers is yet suitable for clinical decision making and most still require biopsies for their evaluation. 


\section{Alternative ways for tissue sampling}

With brush cytology the whole surface area of the Barrett's mucosa may be sampled using a brush that is advanced through the working channel of the endoscope (fig 3). Compared to random biopsies, which sample only a small part of the Barrett's segment, brush cytology may thus reduce sampling error. In addition, brush cytology may detect relevant abnormalities because dysplastic cells shed easier than normal epithelial cells. Furthermore, brushing a Barrett's oesophagus takes considerable less time than obtaining four-quadrant random biopsies at $2 \mathrm{~cm}$ intervals. The morphological analysis of brush cytology specimen, however, is difficult and in fact inferior to standard histology for surveillance of Barrett's oesophagus. ${ }^{25}$ This can be overcome by using FISH (fluorescent in situ hybridisation): a technique in which small fluorescent DNA probes are used for detection of specific chromosomal abnormalities. These fluorescent DNA probes bind specifically to relevant areas of different chromosomes, which enable identification of amplification or loss of areas known to be important for the development of cancer. The use of FISH has important theoretical advantages: because morphological changes in cells are always preceded by genetic changes, FISH may allow earlier detection of patients at risk for malignancy. The surveillance of these patients can then be intensified whereas the majority of patients with no abnormalities should be candidates for a less intensive surveillance programme. Furthermore, instead of using subjective morphological criteria as in the histological evaluation of biopsies, FISH may result in a more objective outcome measurement. Finally, the fluorescent signals generated by multicolour FISH have a strong signal-to-noise ratio, which may enable automated image analysis.

In a recent study, a high sensitivity was demonstrated to detect relevant histological abnormalities by using multicolour FISH on brush cytology specimens of Barrett's patients with high grade dysplasia and/or early cancer. A DNA probe set specific for the locus specific regions harbouring the p53 and pl6 genes and centromeric probes for the chromosomes 9, -17 , and $-\mathrm{Y}$, were used in patients with Barrett's oesophagus with and without dysplasia as well as control patients. The number of genetic abnormalities increased as dysplasia progressed and invasive disease occurred whereas no FISH abnormalities were shown in control patients. ${ }^{26}$

If brush cytology combined with multicolour FISH is proven to be a valid surveillance technique, less invasive ways

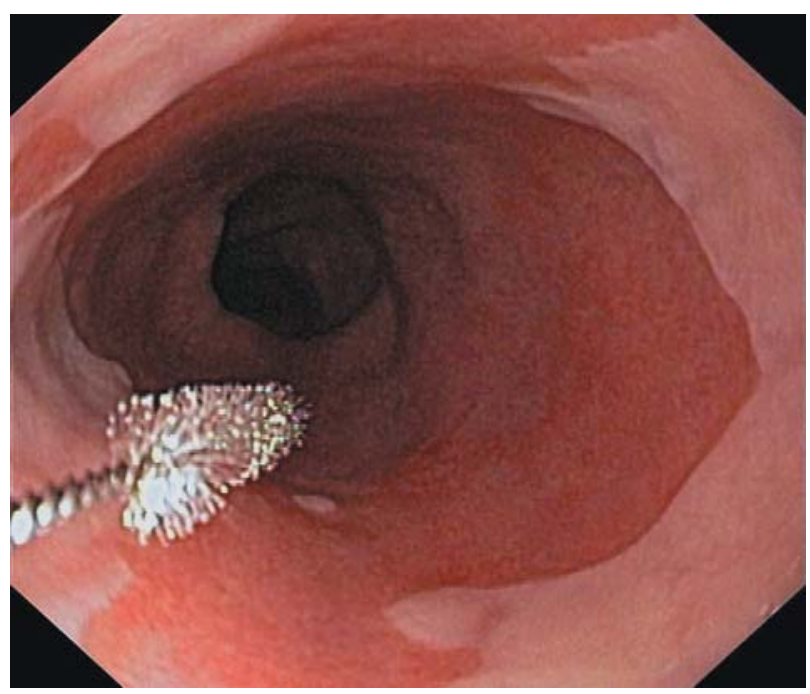

Figure 3 Transendoscopic brush cytology of a Barrett's oesophagus. to obtain a brush specimen may be considered. Transoral and transnasal brushes are being developed that may further improve the cost effectiveness and patients' acceptance of Barrett's surveillance.

\section{CONCLUSION}

Future developments may improve the cost effectiveness of Barrett's surveillance. Less invasive tests may lead to lower cost and higher patients' compliance. The use of molecular markers may enable risk stratification allowing time and efforts to be invested in patients who are truly at risk for malignant degeneration. New endoscopic imaging techniques will improve the detection of relevant lesions in these patients enabling their endoscopic treatment.

The ultimate surveillance technique for Barrett's patients, however, involves a simple blood test for either a genetic or a proteomic abnormality. High throughput cancer discovery techniques combined with advanced bioinformatics tools may lead to the discovery of genomic and proteomic profiles specific for Barrett's patients at risk for developing cancer. ${ }^{27}$

\section{Authors' affiliations}

J J G H M Bergman, G N J Tytgat, Department of Gastroenterology, Academic Medical Center, Meibergdreef 9, 1105 AZ Amsterdam,

Netherlands

\section{REFERENCES}

1 Spechler SJ. Clinical practice. Barrett's esophagus. N Engl J Med 2002;346:836-42.

2 Morales CP, Souza RF, Spechler SJ. Hallmarks of cancer progression in Barrett's oesophagus. Lancet 2002;360:1587-9.

3 Sampliner RE. Practice guidelines on the diagnosis, surveillance, and therapy of Barrett's esophagus. The Practice Parameters Committee of the American College of Gastroenterology. Am J Gastroenterol 1998;93:1028-32.

4 Attwood SE, Morris CD. Who defines Barrett's oesophagus: endoscopist or pathologist? Eur J Gastroenterol Hepatol 2001;13:97-9.

5 Riddell RH. Early detection of neoplasia of the esophagus and gastroesophageal junction. Am J Gastroenterol 1996;91:853-63.

6 Spechler SJ. Columnar-lined esophagus. Definitions. Chest Surg Clin N Am 2002;12:11-13, vii.

7 Montgomery E, Goldblum JR, Greenson JK, et al. Dysplasia as a predictive marker for invasive carcinoma in Barrett esophagus: a follow-up study based on 138 cases from a diagnostic variability study. Hum Pathol $2001 ; 32: 379-88$

8 Guelrud M, Herrera I, Essenfeld H, et al. Enhanced magnification endoscopy: a new technique to identify specialized intestinal metaplasia in Barrett's esophagus. Gastrointest Endosc 2001;53:559-65.

9 Endo T, Awakawa T, Takahashi H, et al. Classification of Barrett's epithelium by magnifying endoscopy. Gastrointest Endosc 2002;55:641-7.

10 Sharma P, Weston AP, Topalovski M, et al. Magnification chromoendoscopy for the detection of intestinal metaplasia and dysplasia in Barrett's oesophagus. Gut 2003;52:24-7.

11 Canto MI, Setrakian S, Willis J, et al. Methylene blue-directed biopsies improve detection of intestinal metaplasia and dysplasia in Barrett's esophagus. Gastrointest Endosc 2000:51:560-8.

12 Dave U, Shousha S, Westaby D. Methylene blue staining: is it really useful in Barrett's esophagus? Gastrointest Endosc 2001;53:333-5.

13 Kara MA, Bergman JJ, Fockens P, et al. Narrow band imaging for mucosal pattern recognition in Barrett's esophagus. Presented at Digestive Disease Week [abstract]. Gastroenterology 2004;126:A50.

14 Panjehpour M, Overholt BF, Vo-Dinh T, et al. Endoscopic fluorescence detection of high-grade dysplasia in Barrett's esophagus. Gastroenterology 1996;111:93-101.

15 Georgakoudi I, Jacobson BC, Van Dam J, et al. Fluorescence, reflectance, and light-scattering spectroscopy for evaluating dysplasia in patients with Barrett's esophagus. Gastroenterology 2001;120:1620-9.

16 Haringsma J, Tytgat GN, Yano H, et al. Autofluorescence endoscopy: feasibility of detection of $\mathrm{Gl}$ neoplasms unapparent to white light endoscopy with an evolving technology. Gastrointest Endosc 2001;53:642-50.

17 Bergman JJ, Kara MA, Smits ME, et al. A randomised cross-over study comparing Light-Induced Fluorescence Endoscopy (LIFE) with Standard Endoscopy (SE) for detection of early neoplasia in Barrett's Esophagus (BE). Presented at Digestive Disease Week [abstract]. Gastroenterology 2003; 124(Suppl 1):A49

18 Li XD, Boppart SA, Van Dam J, et al. Optical coherence tomography: advanced technology for the endoscopic imaging of Barrett's esophagus. Endoscopy 2000;32:921-30.

19 Dacosta RS, Wilson BC, Marcon NE. New optical technologies for earlier endoscopic diagnosis of premalignant gastrointestinal lesions. J Gastroenterol Hepatol 2002; 17(Suppl):S85-104. 
20 Krishnadath KK, Reid BJ, Wang KK. Biomarkers in Barrett esophagus. Mayo Clin Proc $2001 ; 76: 438-46$.

21 Polkowski W, van Lanschot JJ, Kate FJ, et al. The value of p53 and Ki67 as markers for tumour progression in the Barrett's dysplasia-carcinoma sequence. Surg Oncol 1995:4:163-71.

22 Buskens CJ, Van Rees BP, Sivula A, et al. Prognostic significance of elevated cyclooxygenase 2 expression in patients with adenocarcinoma of the esophagus. Gastroenterology 2002;122:1800-7.

23 Tselepis C, Perry I, Dawson C, et al. Tumour necrosis factor-alpha in Barrett's oesophagus: a potential novel mechanism of action. Oncogene 2002;21:6071-81.
24 Haggitt RC, Reid BJ, Rabinovitch PS, et al. Barrett's esophagus. Correlation between mucin histochemistry, flow cytometry, and histologic diagnosis for predicting increased cancer risk. Am J Pathol 1988;131:53-61.

25 Falk GW, Chittajallu R, Goldblum JR, et al. Surveillance of patients with Barrett's esophagus for dysplasia and cancer with balloon cytology. Gastroenterology 1997; 112:1787-97.

26 Krishnadath KK, Liu W, Sebo TJ, et al. Detection of cytogenetic abnormalities by multicolor FISH in brush cytology specimens of Barrett's esophagus. In press 2004.

27 Ardekani AM, Liotta LA, Petricoin EF. Clinical potential of proteonomics in the diagnosis of ovarian cancer. Expert Rev Mol Diagn 2002;2:312-20. 